\title{
Automatic Leak Detection in Buried Plastic Pipes of Water Supply Networks by Means of Vibration Measurements
}

\author{
Alberto Martini, ${ }^{1}$ Marco Troncossi, ${ }^{1,2}$ and Alessandro Rivola ${ }^{1,2}$ \\ ${ }^{1}$ Advanced Applications in Mechanical Engineering \& Materials Technology, University of Bologna, Viale del Risorgimento 2, \\ 40136 Bologna, Italy \\ ${ }^{2}$ Department of Engineering for Industry, University of Bologna, Viale del Risorgimento 2, 40136 Bologna, Italy
}

Correspondence should be addressed to Alberto Martini; alberto.martini6@unibo.it

Received 27 October 2014; Accepted 5 January 2015

Academic Editor: Kenneth J. Loh

Copyright (C) 2015 Alberto Martini et al. This is an open access article distributed under the Creative Commons Attribution License, which permits unrestricted use, distribution, and reproduction in any medium, provided the original work is properly cited.

The implementation of strategies for controlling water leaks is essential in order to reduce losses affecting distribution networks of drinking water. This paper focuses on leak detection by using vibration monitoring techniques. The long-term goal is the development of a system for automatic early detection of burst leaks in service pipes. An experimental campaign was started to measure vibrations transmitted along water pipes by real burst leaks occurring in actual water supply networks. The first experimental data were used for assessing the leak detection performance of a prototypal algorithm based on the calculation of the standard deviation of acceleration signals. The experimental campaign is here described and discussed. The proposed algorithm, enhanced by means of proper signal filtering techniques, was successfully tested on all monitored leaks, thus proving effective for leak detection purpose.

\section{Introduction}

The efficiency of water distribution networks represents a critical issue for all utilities involved in drinking water supply. Available reports $[1,2]$ show that the amount of real (physical) water losses may exceed $30 \%$ of the input volume. Real losses are given by both background leaks (very small leaks occurring at storage tanks or pipe joints and fittings) and burst leaks (resulting from pipe holes and damages). While the former ones are associated with the normal system functioning and cannot be reduced under the limit commonly known as Unavoidable Annual Real Losses, the latter ones are considered potentially recoverable losses. Since losses of drinking water represent a very high cost, a danger for the public health (the leaking flow may damage the foundations of buildings and roads, and contamination by pollutants may occur) and a waste of a limited resource, adopting proper policies for managing burst leaks appears essential.

A number of strategies and technologies have been proposed for implementing leak detection and repair programs.
As for leak detection (namely, discovering the presence of losses in pipeline networks) utilities are getting good results by adopting District Metered Areas (DMA) management [3]. An alternative and still largely adopted approach (although expensive and time consuming) is represented by periodic acoustic surveys for leak detection purpose. Indeed active burst leaks are usually sources of noise that propagates along pipelines and through the ground $[4,5]$. Permanent optimized grids of noise loggers for continuously monitoring the entire water supply network may be also adopted [6], although their economic viability and leak detection effectiveness are not guaranteed [7].

Most of the methods and equipment proposed for leak location (i.e., precisely pinpointing leaks in pipelines) are based on the measurement of leak-related vibro-acoustic phenomena as well. The use of hydrophones and accelerometers for detecting, respectively, fluid-borne and structureborn waves with low-frequencies (i.e., well below the pipe ring frequency, typically below $1 \mathrm{kHz}$ ) generated by leaks and propagating along pipes [8-10] was investigated by numerous studies $[4,6,7,11-15]$. Works dealing with the monitoring 


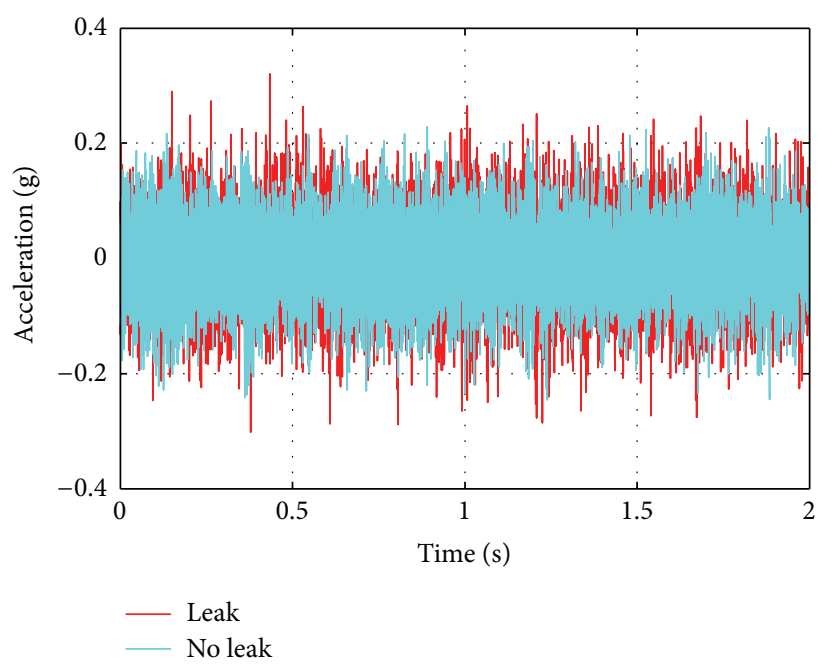

(a)

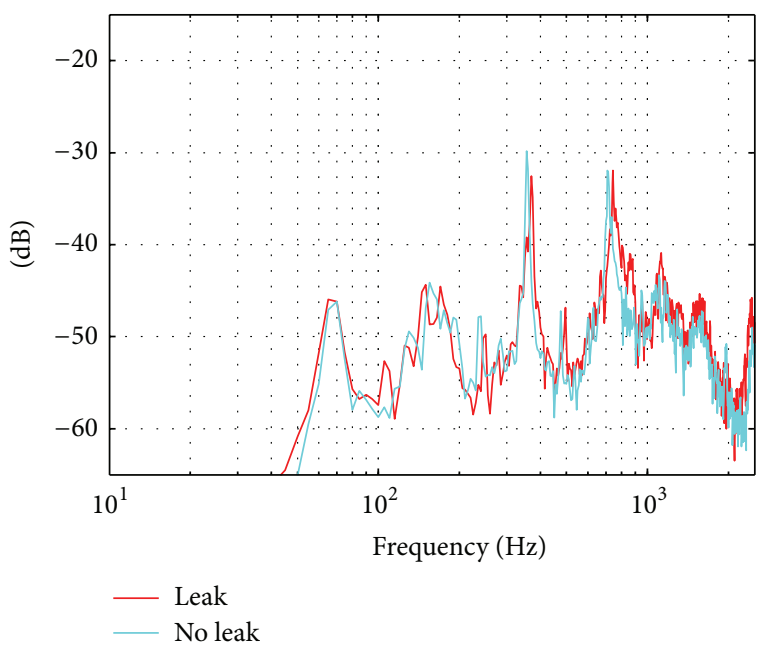

(b)

FIgURE 1: Preliminary vibration measurements with water consumption: (a) time domain, (b) frequency domain.

of Acoustic Emissions (AE, namely, high-frequency elastic waves up to $0.1-1 \mathrm{MHz}$, which may be also generated by active leaks) are available in the literature [16-19]. Other methods and technologies, related or not to vibro-acoustic phenomena (e.g., exploitation of hydraulic transients, thermography, ground-penetrating radar, tracer gas technique, and magnetic fields), were also proposed [13, 20-24]. In practical applications, leak pinpointing is performed by most utilities with common equipment based on vibro-acoustic transducers, such as listening devices (namely, geophones and listening rods, whose efficiency largely depends on the operator skills) and noise correlators (which automatically pinpoint leaks by means of signal correlation techniques).

The research focuses on the detection of burst leaks occurring in service connections (i.e., small diameter pipes connecting customers to the water mains). These leaks frequently present low rates of the leaking flow, and they are therefore characterized by long awareness periods (namely, the time from the burst occurrence to its detection). Consequently their total runtime (i.e., the total period to the burst repair) tends to be rather long, thus leading to overall losses almost comparable to those of large leaks occurring in water mains (which are generally much more rapidly detected or reported). In addition, the increasing use of plastic pipes in water supply networks further contributes to hinder the detection of such leaks. Indeed, problems concerning the significant attenuation of vibro-acoustic phenomena in plastic pipes are known [13], even if the detection of water leaks still appears achievable $[7,14]$.

This work deals with the use of vibration monitoring tools for the detection of burst leaks in water pipelines. The long-term project planned by the multiutility Hera SpA (Bologna, Italy) aims at developing a device for the automatic early detection of unreported bursts occurring in customers' service connections running from the mains to the users' metering point [25]. Indeed water losses related to service pipes represent a significant percentage of the total losses affecting the water distribution network managed by the utility. Nonetheless, to the authors' best knowledge, only few works dealt with this topic and no studies on small diameter plastic pipes are available in the literature; thus specific investigations are advisable. The system is meant to be installed near the water meter and to autonomously detect and report the presence of leaks. This implies a certain detection algorithm to be run on-board. Low cost is one of the main design requirements of the final device, since a large number of devices will be needed for covering the entire network. In particular, in order to work with limited computational resources (thus lowering hardware costs), the final detection algorithm will require only simple operations to be executed. The system is expected to significantly reduce the awareness time of a large amount of water leaks, thus globally cutting the costs associated with non-revenue water.

Preliminary experimental tests had been performed on both a test rig and one actual service pipe of the water distribution network, by considering artificially induced leaks [26]. The investigation confirmed vibration monitoring as an effective tool for leak detection purpose, provided that no water flow induced by the customers' water consumption is present inside the monitored pipe. In such an instance, indeed, even very small values of the flow rate cause high vibration levels which cover the effects of the leak, thus making the detection impossible (Figure 1). The experimental data permitted to define a prototypal detection algorithm capable of automatically distinguish between leaking and non-leaking conditions [26]. The implemented algorithm uses the signal standard deviation (STD) as a metric to detect the increment of vibration levels induced by the leaking water flow.

A broader experimental campaign was started for collecting vibration data from real burst leaks detected and repaired in actual service pipes of the water distribution network managed by the utility. This paper describes the experimental campaign and presents the first results. In particular, 


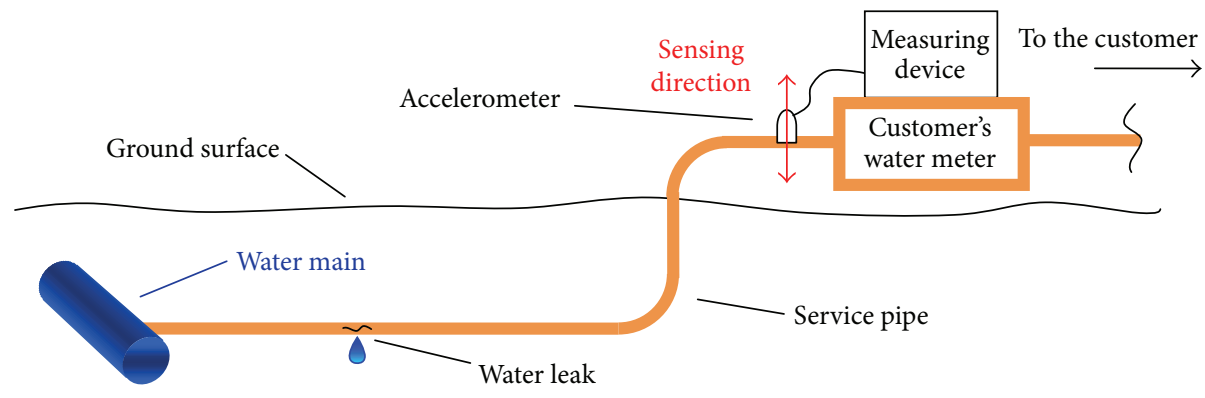

(a)

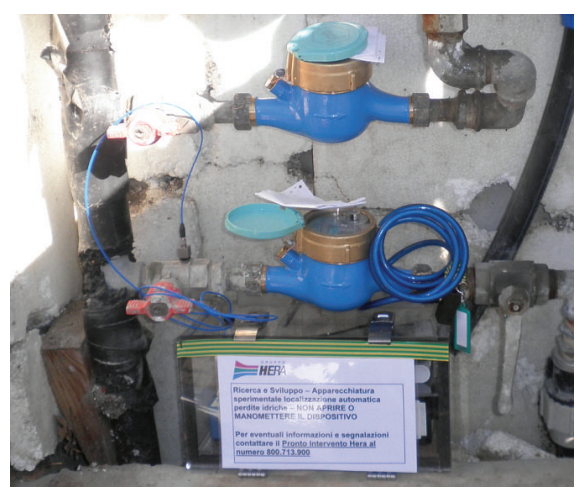

(b)

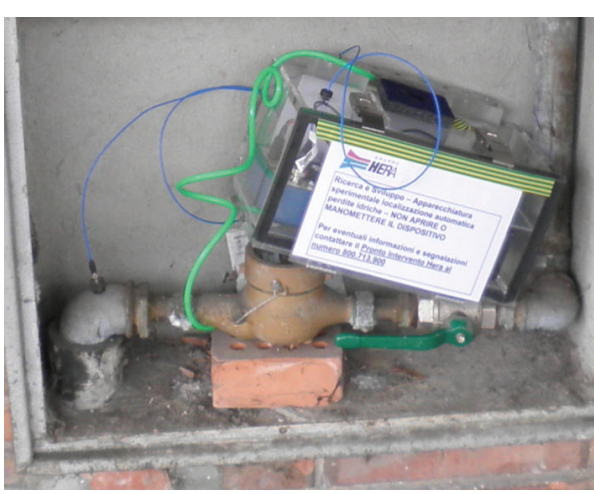

(c)

FIGURE 2: Pictures of the measurement setup for two monitored sites.

the performance of the proposed detection algorithm is assessed and discussed. Possible signal processing techniques for enhancing the algorithm sensitivity and robustness are also investigated.

\section{Experimental Setup and Data Processing}

The experimental campaign was meant for investigating vibrations generated on pipes by real burst leaks occurring in service connections. In particular, the objective was performing measurements in both leaking and non-leaking conditions for each monitored pipe, to assess the differences between the signals characterizing each condition.

The custom acquisition devices arranged in the preliminary phase of the research [26] were adopted for the measurements. Each unit presents the following configuration. Vibrations are detected by a commercial IEPE accelerometer (sensitivity $1 \mathrm{~V} / \mathrm{g}$ ). A signal conditioner powers the sensor and provides a signal gain of 100 . The conditioned signal is acquired and recorded by a GigalogF datalogger with modified firmware (sampling frequency $3300 \mathrm{~Hz}$ ). A battery pack powers the system; thus external power supply is not required.

The majority of the service pipes of the network, as the ones monitored in this study, lie very close or even under roads featuring not negligible traffic; hence measurements may be affected by significant perturbations unrelated to leaks. In order to reduce the incidence of such kind of phenomena, measurements were performed by night (namely, after midnight) when the environmental noise related to human activities is limited (including the demand of water by customers, which is also detrimental for leak detection operations). This strategy is a commonly adopted for leak detection surveys. The possible delayed detection of leaks occurring in the daytime is considered acceptable, since a remarkable reduction of the awareness time is still reasonably expected.

As a pilot study, all measurements were performed on leaks already detected or reported. Firstly, acquisitions of vibration signals were performed in leaking condition, before the repair work. Then the undamaged condition was restored and further measurements were carried out in non-leaking state. The measurement protocol, whose parameters were defined on the basis of the results provided by preliminary tests, is described hereafter.

(i) The maintenance team preliminarily checks the leak and installs the measuring unit near the customer's water meter (Figure 2). The accelerometer is mounted on the connection pipe (or on the metal fittings/valves installed in the last portion of the pipe near the water meter) with radial sensing direction, by means of petroleum wax (Figure 2(a)). The main characteristics of the monitored leak and of the corresponding measurement setup are noted (namely, the distance between the pipe damage and the transducer location, the type of customer, the specific measuring unit, and a rough estimation of the flow rate). 
(ii) The measuring device automatically performs measurements during night, starting at 00:30. Sixty acquisitions per night (one record every 5 minutes with duration of $10 \mathrm{~s}$ each) are carried out for some consecutive nights. Since vibration phenomena related to water leaks exhibit stationary signals, the acquisition of relatively short time histories is sufficient for assessing the pipe status, as also confirmed by the preliminary tests (whereas longer acquisitions may result in a higher incidence of transient perturbations related to the environmental noise).

(iii) The maintenance team repairs the burst by substituting the whole connection pipe and restores the undamaged condition. During the maintenance work, the sensor setup is not modified (i.e., the accelerometer is never removed from the initial measuring location).

(iv) Acquisitions in non-leaking condition are carried out for several consecutive nights after the repair, until the unit is removed (generally after about a week). The recorded data are then extracted for the analysis. At this stage of the research both the algorithm execution and further signal processing are performed offboard.

It should be noticed that while higher leak-related vibrations would be expected along the pipe axis [9], the measurement of axial vibrations in actual service pipes is often impractical since only its very last portion next to the flow meter is usually accessible (Figure 2(c)) and significant additional modifications would be required. The results presented hereafter were obtained by installing all transducers with radial sensing direction.

Although measurements did not follow the natural evolution of the leak, the adopted procedure permitted to obtain data from real leaks occurring in different districts of the entire water distribution network managed by the utility, thus possibly taking into account most of the boundary and functioning conditions characterizing the network. Data related to thirteen different burst leaks were collected.

The soundness of the acquired datasets (simply referred to as DS hereafter) was firstly checked in order to investigate the presence of possibly unexpected signal features and to assess the algorithm capabilities of dealing with such features. Then the data were processed with the prototypal detection algorithm, in order to test its effectiveness. The algorithm uses the STD as a parameter to monitor the increment in the pipe vibration levels that is experienced when a water flow is leaking from a damaged pipe. This strategy had been proven effective for leak detection purpose by the mentioned preliminary tests [26]. In particular, the following operations are performed.

(i) The signal STD is computed for all records collected in a night, thus obtaining 60 STD values for each night of measurements. The algorithm is directly run on the raw signals.

(ii) A proper index characterizing each night of acquisition (hereafter referred to as Monitoring Index (MI)) is

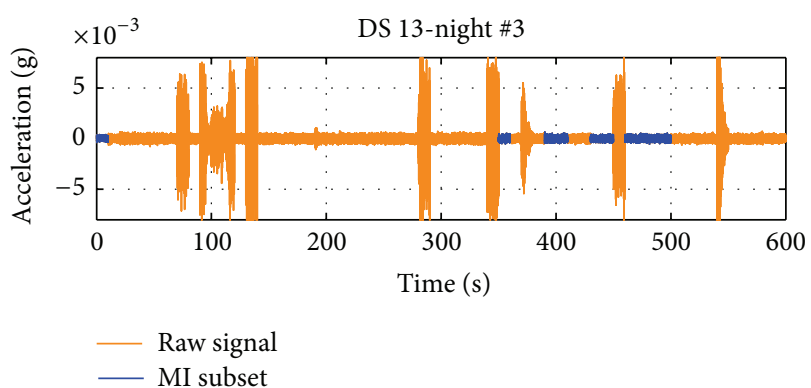

(a)

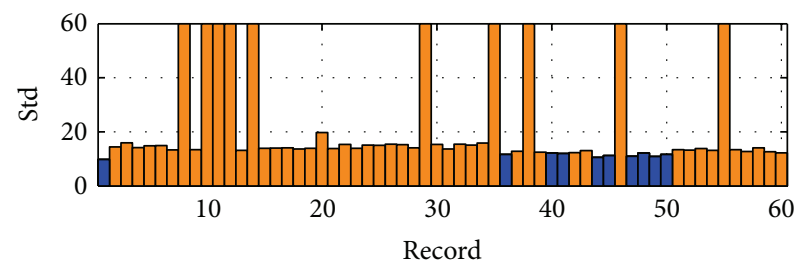

(b)

FIgURE 3: (a) Complete time history and (b) STD values for night \#3 of dataset DS 13; the subset considered for the MI computation is highlighted in blue.

determined from a subset of the corresponding STD values (Figure 3(b)). The MI of the $j$ th night is defined as

$$
\mathrm{MI}_{j}=\operatorname{mean}\left(\bar{\sigma}_{j, 10}\right) \text {, }
$$

where $\bar{\sigma}_{j, 10}$ is the vector of the 10 lowest STD values of the $j$ th night, taken from the vector of all STD values of the same night, $\bar{\sigma}_{j, 60}$ (i.e., $\bar{\sigma}_{j, 10} \subset \bar{\sigma}_{j, 60}$ ). Therefore the MI is computed by considering only a reduced portion (with duration of $100 \mathrm{~s}$ ) of the whole record (lasting $600 \mathrm{~s}$ ) available for each night (Figure 3). The selection of a reduced subset permits to neglect signals affected by perturbations unrelated to the leaks (clearly visible in Figure 3(a)), so that only stationary signals are taken into account for assessing the pipe status.

(iii) Each $\mathrm{MI}_{j}$ value is compared with a threshold level defined on the basis of measurements characterizing the non-leaking status (performed when the monitoring system is installed on the monitored pipe). The presence of an active leak causes an increment in the pipe vibration levels and therefore makes the MI value rise. If the $\mathrm{MI}_{j}$ crosses the threshold, the system gives a warning that alerts the maintenance teams so that leak pinpointing and repair operations can be started.

As for the experimental data considered in this study, the presence of leaks is known a priori and the datasets are characterized by a very limited number of nights. Therefore, only for these first experimental tests, the MI of the nights in leaking condition are directly compared with the MI values of the non-leaking status, without defining a threshold level. At this stage a further index, referred to as Monitoring Index 
TABLE 1: Characteristics of the monitored leaks and related measurements and results of the algorithm applied to raw signals and band-pass filtered signals.

\begin{tabular}{lcccccccc}
\hline Dataset & Flow rate & Distance $[\mathrm{m}]$ & $\begin{array}{c}\text { nr. night } \\
\text { leak }\end{array}$ & $\begin{array}{c}\text { nr. night } \\
\text { no-leak }\end{array}$ & \multicolumn{2}{c}{ Raw signals } & \multicolumn{2}{c}{ Filtered signals } \\
Detection & MIE & Detection & MIE \\
\hline DS 1 & Low & 1.6 & 2 & 6 & Yes & 217.55 & Yes & 174.95 \\
DS 2 & Low & 9.8 & 2 & 4 & No & 0 & Yes & 1.72 \\
DS 3 & Medium & 10 & 2 & 3 & Yes & 14.34 & Yes & 28.05 \\
DS 4 & Low & 2 & 2 & 5 & Yes & 51.03 & Yes & 38.41 \\
DS 5 & Low & 4 & 2 & 4 & Yes & 2.04 & Yes & 2.25 \\
DS 6 & Low & 0.5 & 2 & 5 & Yes & 251.29 & Yes & 196.53 \\
DS 7 & High & 3 & 1 & 7 & Yes & 23.79 & Yes & 22.57 \\
DS 8 & Low & 1 & 4 & 3 & Yes & 19.54 & Yes & 29.06 \\
DS 9 & Low & 0.5 & 1 & 7 & Yes & 412.18 & Yes & 650.99 \\
DS 10 & Low & 3 & 1 & 6 & No & 0 & Yes & 2.25 \\
DS 11 & Low & 3.5 & 2 & 6 & No & 0 & Yes & 1.79 \\
DS 12 & Medium & 7 & 1 & 7 & Yes & 26.64 & Yes & 60.83 \\
DS 13 & Low & 6 & 2 & 4 & Yes & 2.42 & Yes & 7.55 \\
\hline
\end{tabular}

Efficiency (MIE), was computed for each dataset, in order to better evaluate the algorithm efficiency in terms of sensitivity to the leak. The MIE of a dataset was defined as

$$
\mathrm{MIE}=\frac{\mathrm{MI}_{\max }}{\operatorname{mean}\left(\mathrm{MI}_{j, \text { no-leak }}\right)},
$$

where $\mathrm{MI}_{\max }$ is the maximum $\mathrm{MI}$ of the dataset and $\mathrm{MI}_{j, \text { no-leak }}$ is the MI of the $j$ th night in non-leaking state. This definition implicitly assumes that the maximum occurs for measurements in leaking condition; that is, that the leak is correctly detected. Detecting leaks with satisfactory reliability is a primary requirement. Indeed a low sensitivity may cause false detections, thus resulting in an unaffordable increment in leak management costs due to unnecessary operations of maintenance teams.

Additional analyses were carried out for possibly enhancing the performance of the detection algorithm. Only rather basic processing techniques were considered, in order to meet the requirements of the final leak detection system. The raw signals were processed in the time domain for the calculation of some common statistics, namely, RMS value, skewness, and kurtosis. Variants of the prototypal algorithm based on these metrics were tested as possible alternatives. The signature of the water leaks in the frequency domain was also investigated by analyzing the signal power spectrum (PSD).

\section{Results and Discussion}

All the monitored burst leaks were associated with highdensity polyethylene (HDPE) service pipes of small diameter (DN 32). Information concerning the leak flow rate (roughly estimated, by using a three-level scale, as "low," "medium," and "high"), the distance between the leak and the transducer, and the amount of nights before and after the repair (leaking and non-leaking conditions, resp.) in which acquisitions were performed is reported in Table 1 for each dataset. Distance values are rather uniformly distributed in the range $0-10 \mathrm{~m}$, thus making this first database fairly representative of bursts possibly occurring in the network. Almost all leaks present low flow rates. This condition is the most useful for properly verifying the algorithm, since high values would make the detection largely easier.

The analysis of the general characteristics of the acquired signals shows that measurements are partially affected by perturbations unrelated to the leaks. The complete time histories related to nights \#1 and \#3 of DS 5 are reported in Figures 4(a) and 4(b), as examples. The STD values computed for the same signals are shown in Figures 4(c) and 4(d). The corresponding spectrograms, obtained by computing the Short-Time Fourier Transform (using a Hann window of length of 4096 samples without overlap), are also shown in Figures $4(\mathrm{e})$ and $4(\mathrm{f})$.

The behavior of the signals is consistent with the results of preliminary tests [26]. Leak-related vibrations exhibit stationary signals which are all characterized by the same spectral lines (which therefore appear as system resonances, i.e., horizontal lines in the spectrogram). The background noise is characterized by stationary signals as well.

Two different kinds of perturbations can be observed. The former is represented by transient signals with variable frequency content. For night \#3 of DS 5 such phenomena are clearly visible in the bandwidth $400-1000 \mathrm{~Hz}$, in the time interval 110-170 s (Figure 4(f)). The latter is represented by stationary signals featuring high vibration levels over the entire spectrum. In night $\# 1$ they occur at time $100 \mathrm{~s}$ and in the last part of the time history. In night \#3 they are clearly 


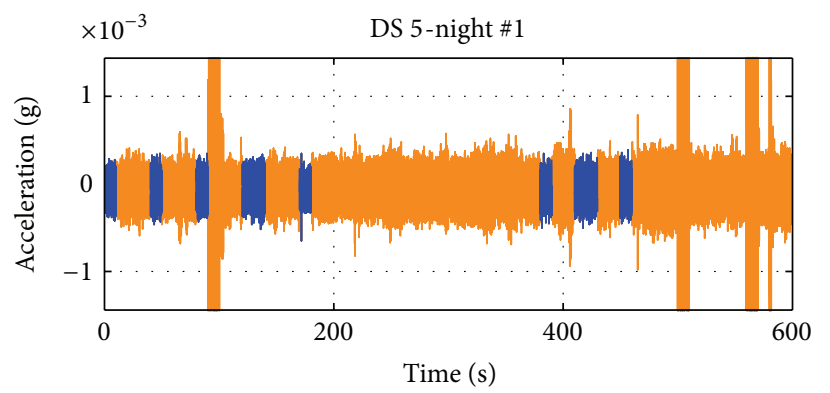

(a)

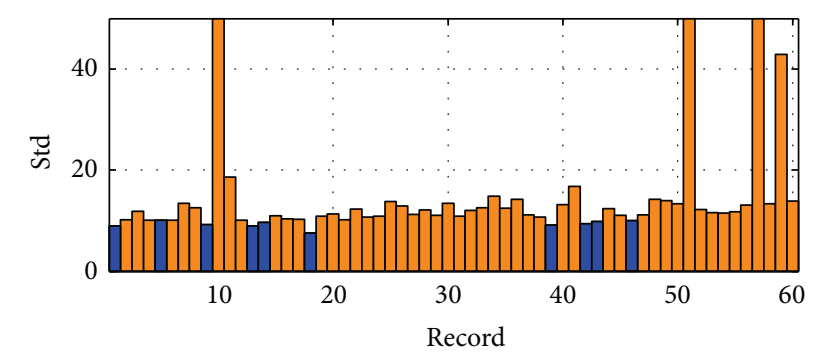

(c)

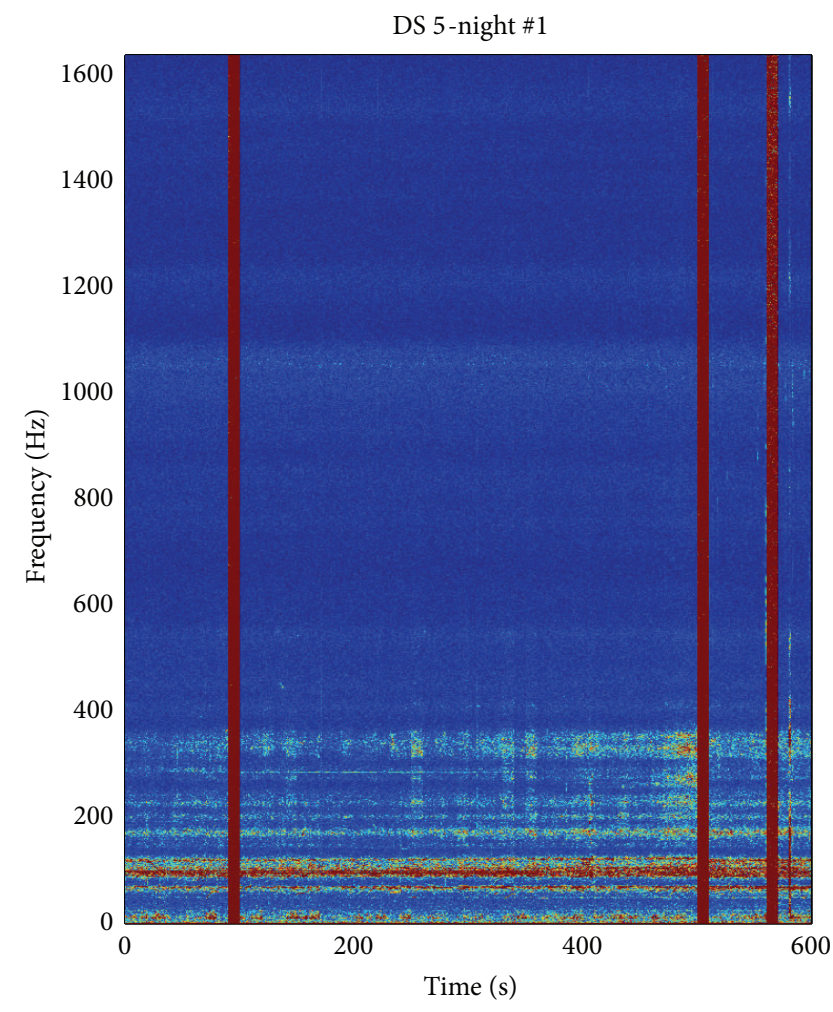

(e)

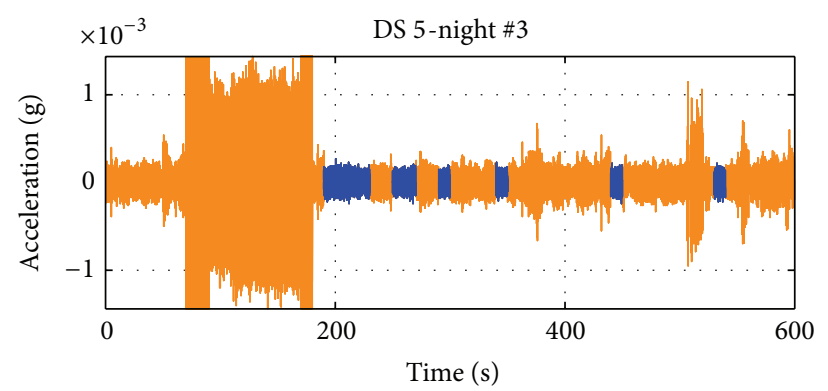

(b)

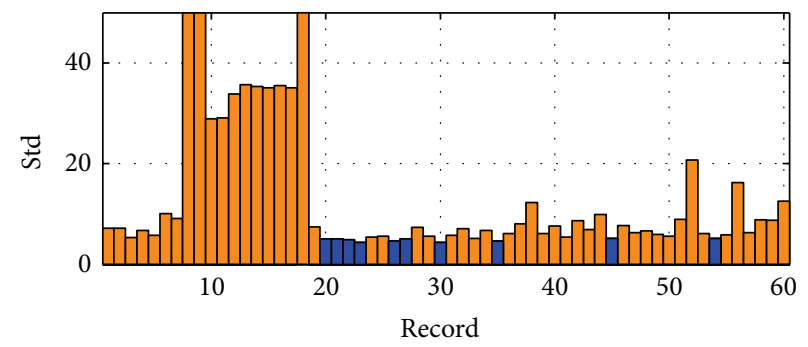

(d)

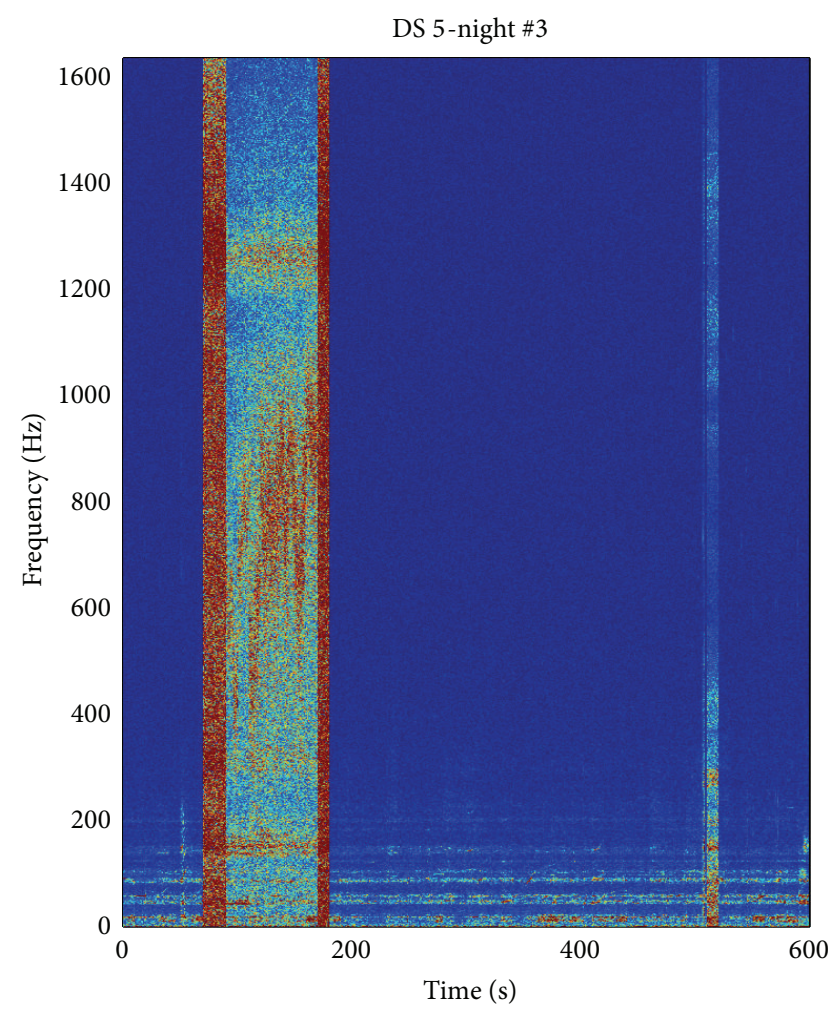

(f)

FigURE 4: Investigation of signals related to nights \#1 and \#3 of dataset DS 5 (a), (b) complete time history; (c), (d) STD values; (e), (f) time-frequency analysis. 


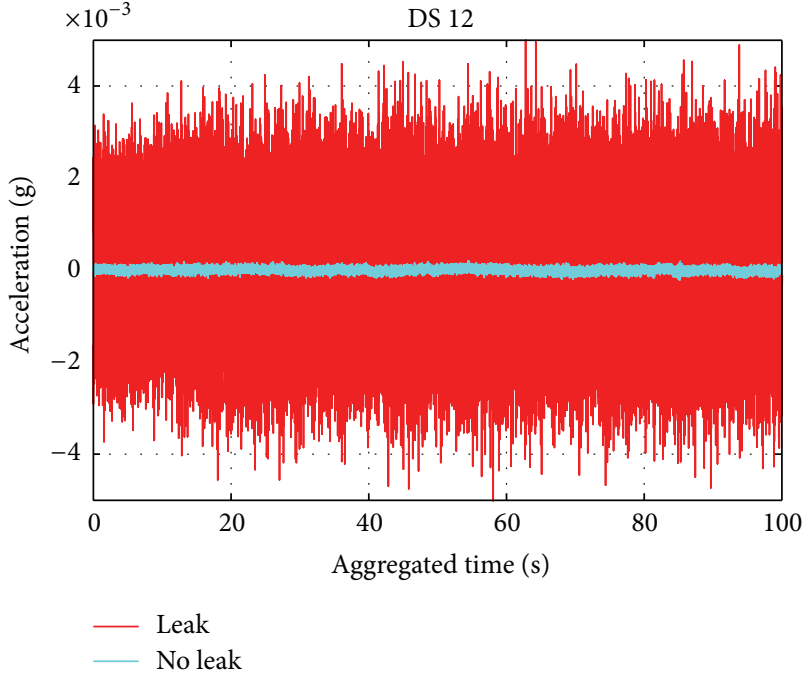

(a)

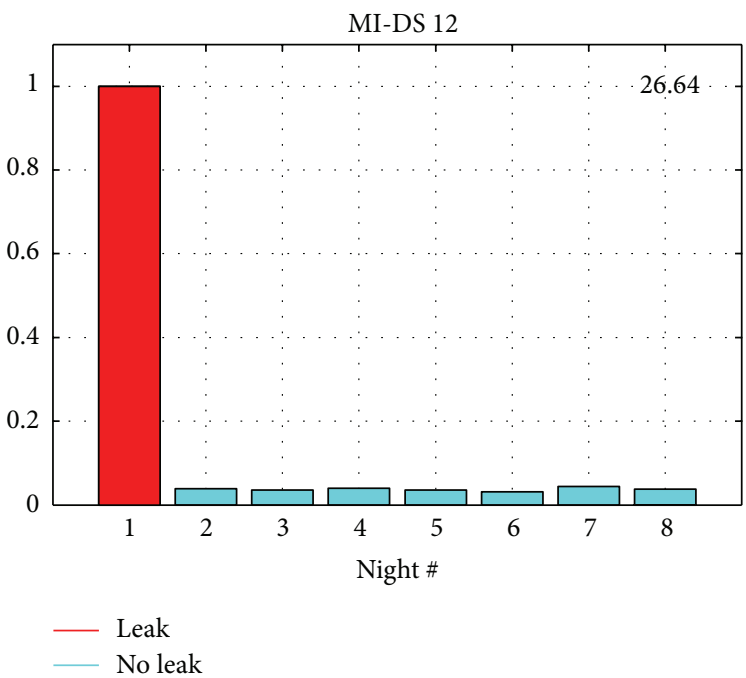

(b)

FIGURE 5: (a) Aggregated time histories and (b) computed MI values of raw signals from dataset DS 12.

visible before and after the transients. Since both kinds of perturbations are associated with a remarkable increment in the vibration levels, they also result in a significant increment of the signal STD. The definition of the reduced subset performed by the algorithm is therefore sufficient for neglecting all acquisitions affected by such phenomena.

The verification of the acquired signals also reveals continuous saturation of the ADC board in leaking condition for datasets DS 1, DS 6, and DS 9, which are basically related to the least distant leaks. Saturation was quite unexpected for leaks featuring low flow rates. Indeed, vibration levels measured in preliminary tests were rather low and the amplification gain was set to 100 in later experiments in order to better exploit the range of the acquisition board. Nonetheless such datasets are processed as well, since the detection algorithm is designed to handle also signals affected by saturation, maximum vibration level characterizing actual leaks being not completely known a priori.

The results obtained by processing all the datasets are summarized in Table 1. In particular the detection result (successful or missed detection), as well as an evaluation of the sensitivity to the leak (provided by the MIE), is reported. A value equal to 0 is assigned to the MIE whenever the leak is not detected. The detection is successful for 10 datasets. In 2 of these cases (DS 5 and, to a certain extent, DS 13) a poor performance is observed, the MIE having a value of about 2 . The leaks exhibiting saturated signals are correctly detected and the efficiency is extremely high, since saturation is obviously associated with a remarkable increment in the signal STD value. In general the results are consistent with the characteristics of the leaks; that is, the worst performances are achieved for leaks distant from the sensor and characterized by a low flow rate.

It is worth noticing that the diagnostic tool is not intended for leak pinpointing. Indeed, once a leak is detected in a specific connection branch, it may be straightforwardly located by maintenance teams by using common equipment (such as correlators or listening devices), since the average length of the service pipes of the water distribution network is about $10 \mathrm{~m}$.

Figure 5(a) shows the acceleration signals concerning two nights (immediately before and after the repair work) of the dataset DS 12, taken as an example for the cases of successful detection. The plot reports the time histories obtained by aggregating the raw signals of 10 acquisitions (namely, those with the lowest STD values, i.e., those processed by the algorithm, as shown in Figure 3). The MI values (normalized to the highest term) obtained for the dataset are shown in detail in Figure 5(b) (values related to the leaking condition are marked in red). The corresponding MIE is reported in the upper-right corner. As predicted, a significant increment in vibration levels associated with the presence of the leak is observed (Figure 5(a)). The MI values rise correspondingly, thus permitting leak detection (Figure 5(b)).

A close examination in the time domain to the five cases characterized by unsatisfactory algorithm performances reveals extremely low vibration levels. Figure 6 reports some time histories concerning two relevant cases, DS 5 and DS 11, taken as examples for the situations of poor performance and missed detection, respectively. The measured accelerations never exceed amplitude of $5 \cdot 10^{-4} \mathrm{~g}$ for both leaking and non-leaking conditions. Such levels make distinguishing the vibrations associated with the leak from the background noise difficult, thus causing problems for the algorithm.

The MI values computed for the examined datasets are shown in detail in Figure 7. As for DS 5 (Figure 7(a)), the MI associated with the two nights in leaking conditions are higher than the other, thus allowing to detect the leak, though with little sensitivity. Results are significantly worse for dataset DS 11, where the MI value obtained for the last night (Figure 7(b)) dominates the other values (which are all comparable), thus preventing the leak from being detected. 


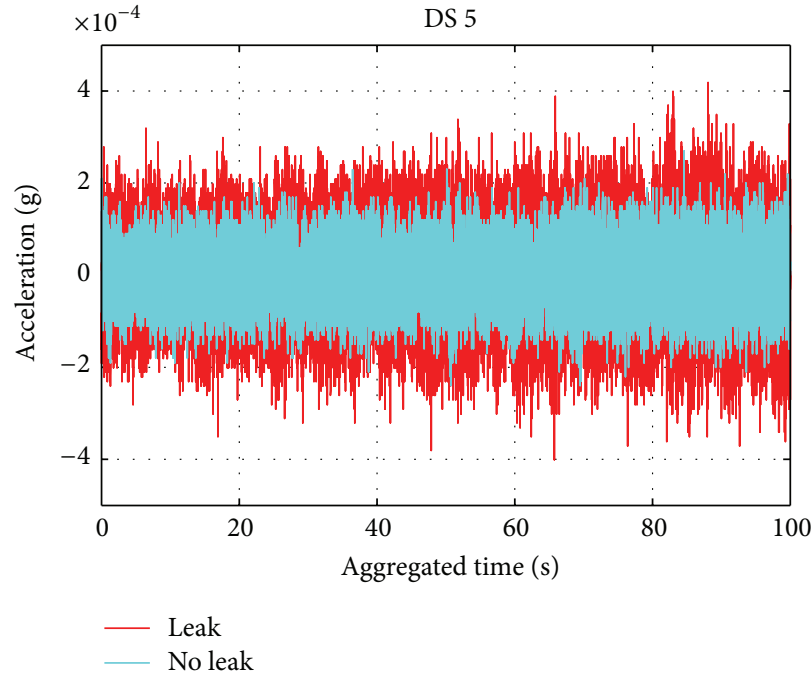

(a)

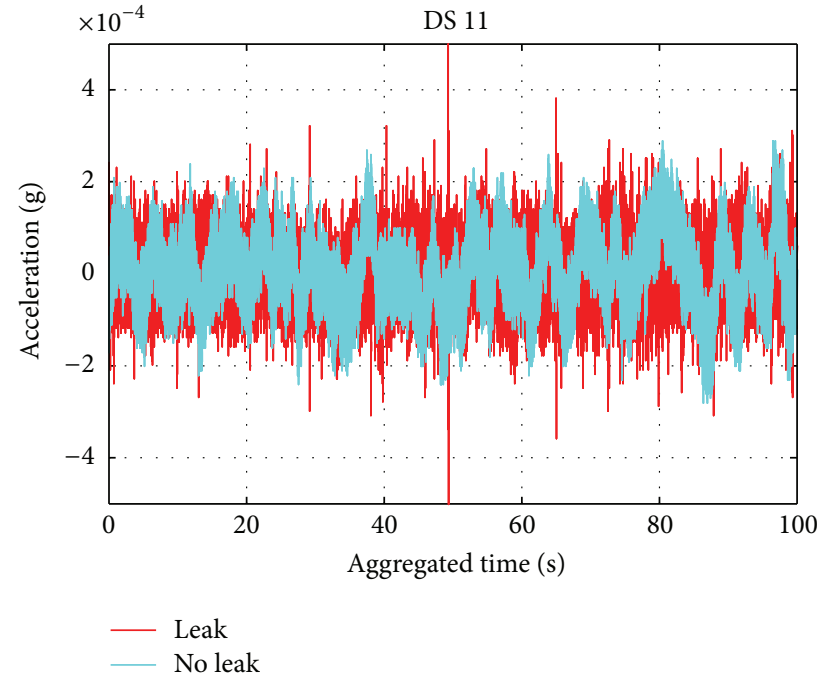

(b)

FIGURE 6: Aggregated time histories of the reduced datasets obtained from (a) nights \#2 (leak) and \#3 (no leak) of DS 5 and (b) nights \#2 (leak) and \#8 (no leak) of DS 11.

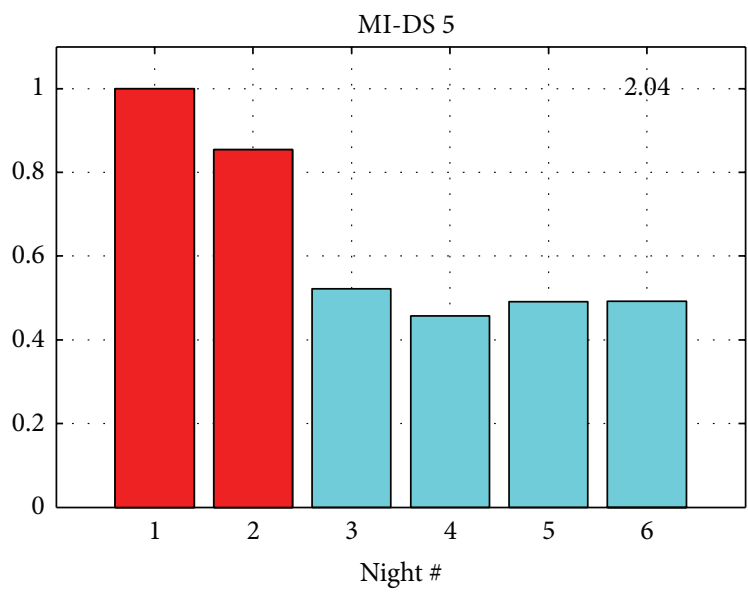

(a)

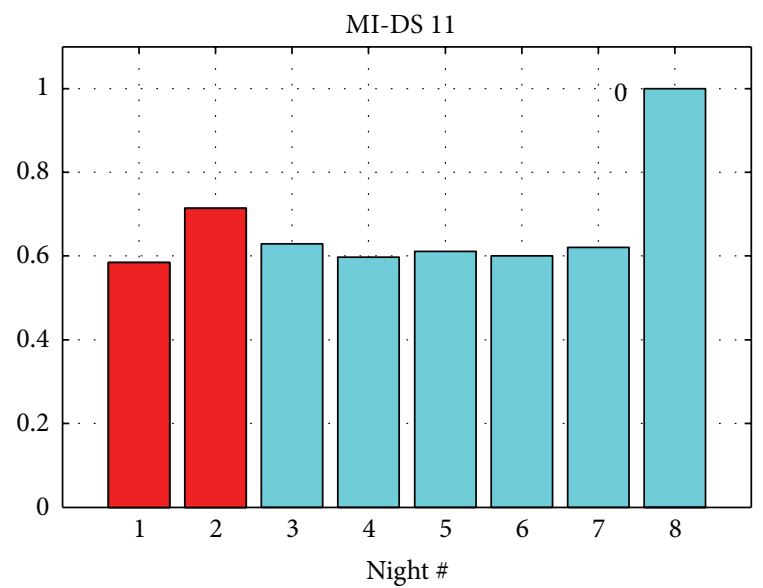

(b)

FIgURE 7: MI computed for datasets (a) DS 5 (poor performance) and (b) DS 11 (missed detection).

The PSDs of the reduced time histories processed by the algorithm are computed for all cases not affected by saturation. Figures 8 and 9 report the comparison between the PSDs concerning, respectively, the nights immediately before and after leak repair, for all analyzed datasets. The spectral analysis reveals that signals recorded in non-leaking conditions are generally dominated by components at relatively low frequencies (below $150 \mathrm{~Hz}$ ). Conversely, active leaks induce vibrations in a wider frequency band. The datasets DS 4 and DS 8 exhibit high vibration levels over the entire spectrum. This behavior is very similar to the effects associated with non-null flow rate condition (induced by customers' water usage) and it is reasonably ascribable to the short distance between the leak and the transducer. The signals related to all the other leaks feature rather different frequency content. As expected, due to the variability of boundary conditions associated with the tested sites, the monitored leaks do not exhibit identical signatures in the frequency domain. However some frequency bands common to all signals associated with active leaks can be identified. Such bands can be exploited for the definition of proper band-pass filters to process the signals before running the algorithm. Different filters were successfully tested. The results provided by the algorithm after the application of a $200-600 \mathrm{~Hz}$ bandpass filter (viz. a 32th order FIR digital filter) are shown in Figure 10 for the datasets DS 5 and DS 11. For both cases an appreciable increment in the algorithm performance is observed. In particular, in the latter dataset the highest MI value is now exhibited for the night preceding the repair (night \#2), whereas the last night of measurements is no 


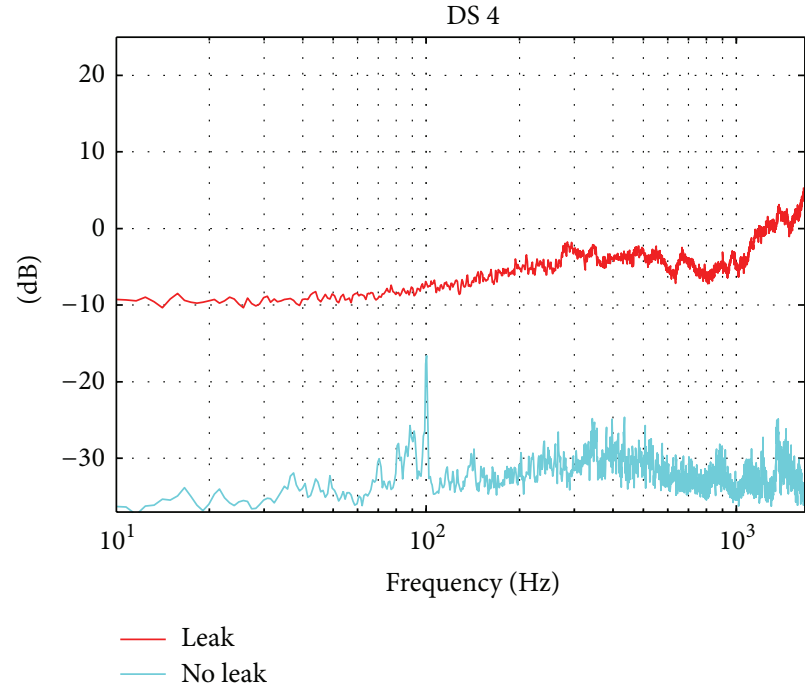

(a)

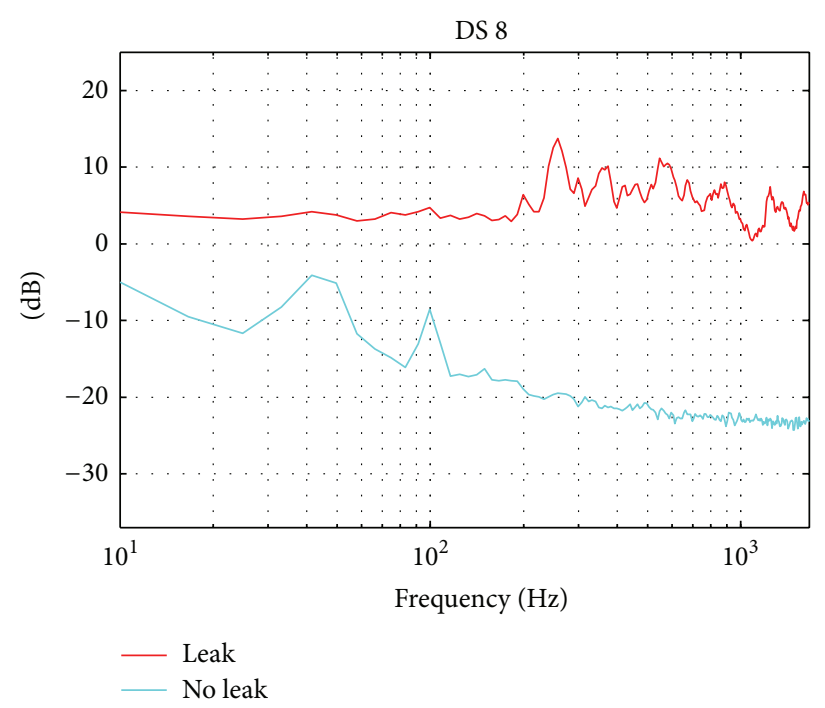

(b)

FIGURE 8: PSD of aggregated signals from datasets (a) DS 4, (b) DS 8.

more affected by any anomaly, thus allowing for the possible detection of the leak, though with a low efficiency.

The results obtained with the same filter for the whole database are summarized in Table 1 . Signals affected by saturation are filtered as well, thus simulating the presence of a band-pass filter in the measurement setup. The algorithm performs remarkably better, since all leaks are detected. In particular, improvements are observed for all datasets previously characterized by poor performances, while all the other cases still exhibit satisfactory results. These results prove band-pass filtering as a potentially profitable solution for enhancing the algorithm, since its detection performance may be improved with a moderate increment of its complexity.

It is worth noticing that the vibration signals analyzed in this paper are characterized by relatively high frequencies, whereas values reported in the literature for burst leaks occurring in plastic pipes are typically below $200 \mathrm{~Hz}[12,14$, 15]. Reasonably the different behavior is ascribable to the small dimensions of pipes monitored in this study, while in the other works larger and longer pipes are considered, even if a deeper assessment of the system vibrational properties would be required.

The investigation of other statistical parameters provided unsatisfactory results. The comparison between the STD values of the reduced subset considered for MI computation and the corresponding skewness and kurtosis values is reported in Figure 11 for DS 7, as example, for both leaking and non-leaking conditions. Such leak can be detected with high efficiency by using STD, whereas no appreciable correlation between the presence of the leak and the trend of the two alternative parameters can be identified. Analogous considerations hold for all examined datasets, including those characterized by poor detection performance of the STDbased algorithm. Hence neither the skewness nor the kurtosis proved suitable for implementing effective variants of the leak detection algorithm.

Conversely the results obtained by using the RMS value were comparable to those provided by the STD-based algorithm. Indeed STD and RMS values coincide for signals characterized by null mean value. In case of low vibration levels, the correct operation of a RMS-based algorithm may result significantly hampered by a signal bias possibly introduced by the measuring setup (behavior occasionally experienced within the experimental campaign). However, if a filter that removes the signal continuous component was applied (such as the proposed band-pass filter), the use of STD or RMS would be equivalent.

\section{Conclusions}

This paper presented a study concerning the detection of burst leaks in water pipes by means of vibration monitoring. An experimental campaign was started for measuring vibration signals associated with real leaks occurring in actual service pipes of a water distribution network. The effectiveness of a prototypal algorithm for leak detection purpose was verified with the first acquisitions performed within the campaign. The algorithm is based on the simple standard deviation computed on raw signals, thus being very simple to be implemented in practice and requiring very limited computational resources to be executed.

The research provided relevant information concerning water leaks in small diameter plastic pipes, a topic which, to the authors' best knowledge, had been only marginally studied in the literature.

The results of the experimental tests were rather satisfactory. The detection of all examined leaks was achieved with acceptable efficiency by means of proper band-pass filters. 


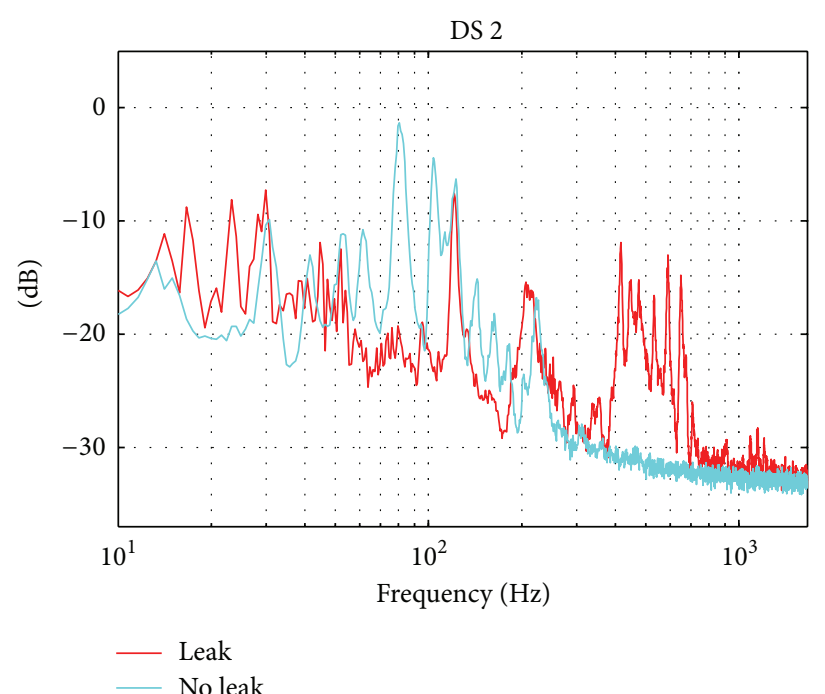

(a)

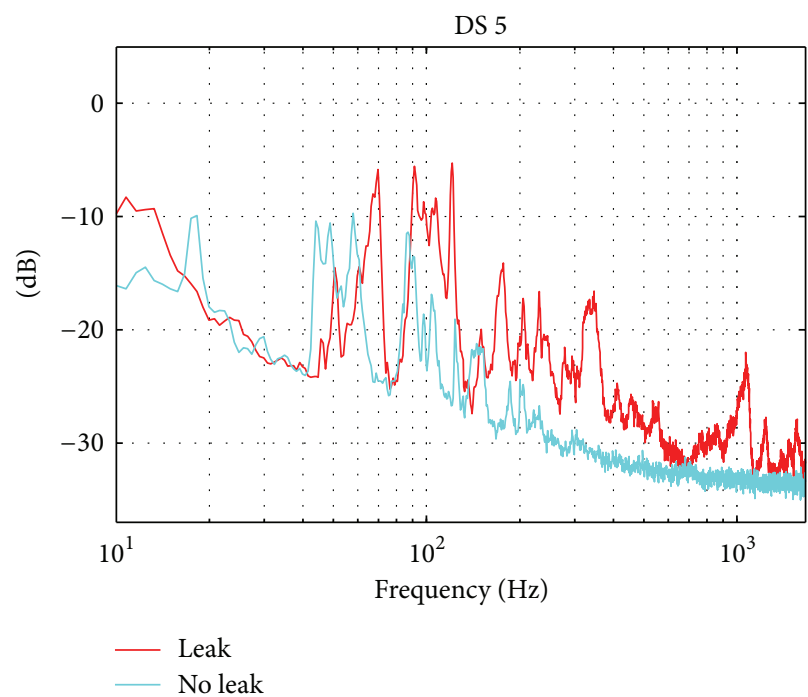

(c)

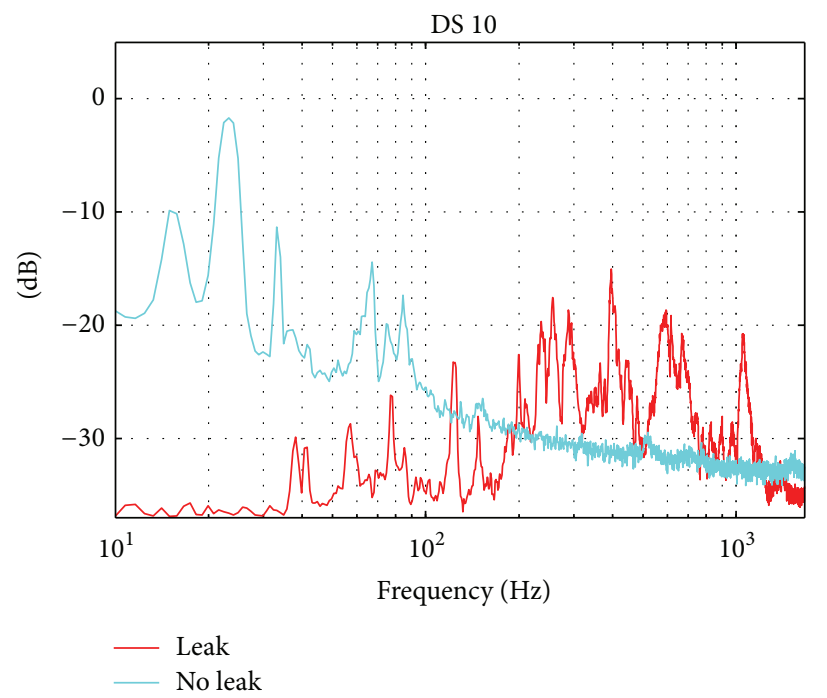

(e)

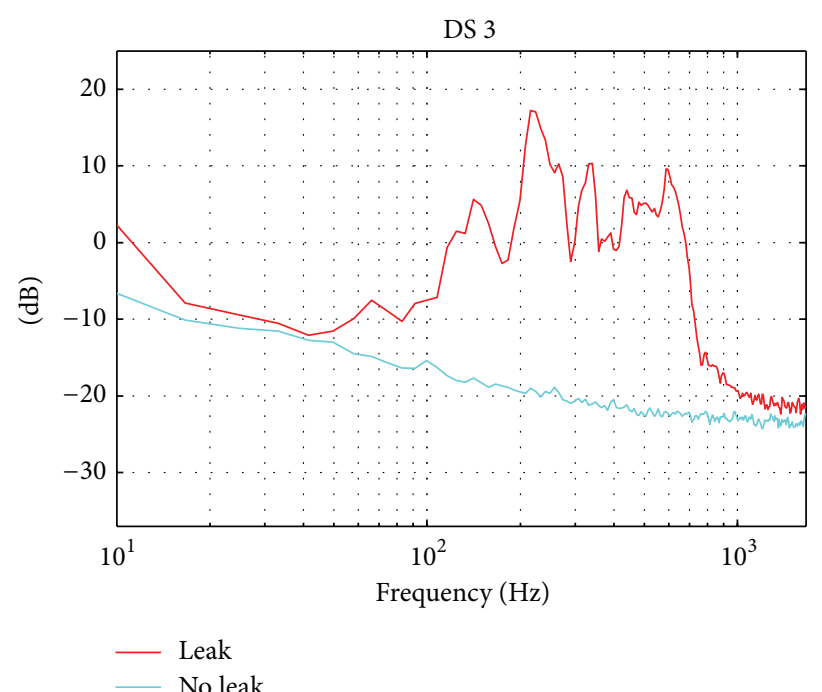

(b)

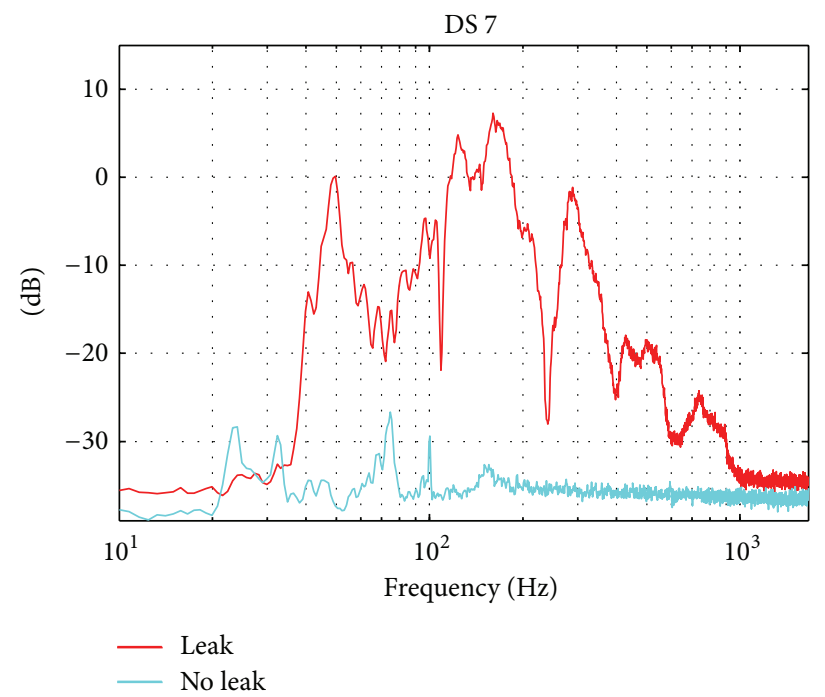

(d)

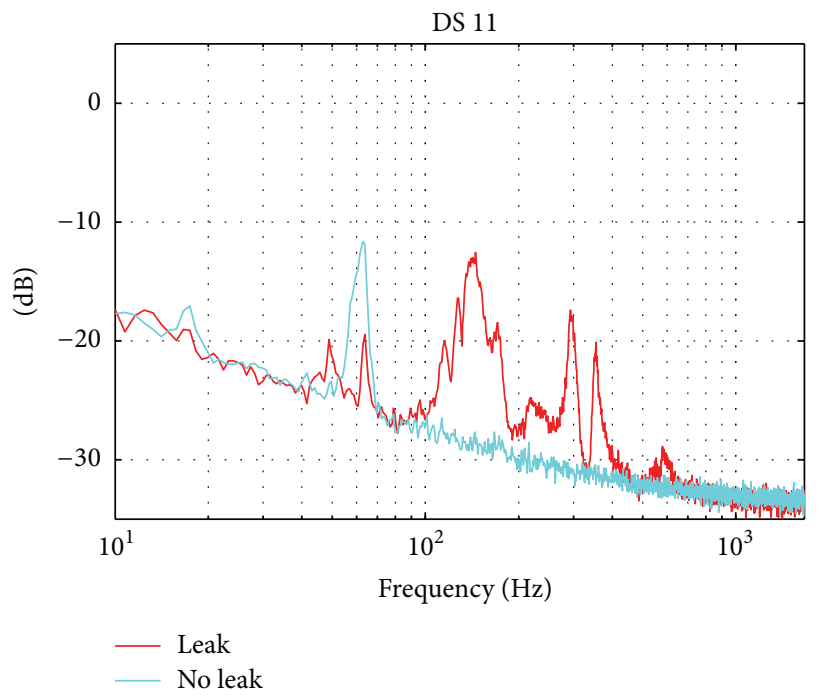

(f)

Figure 9: Continued. 


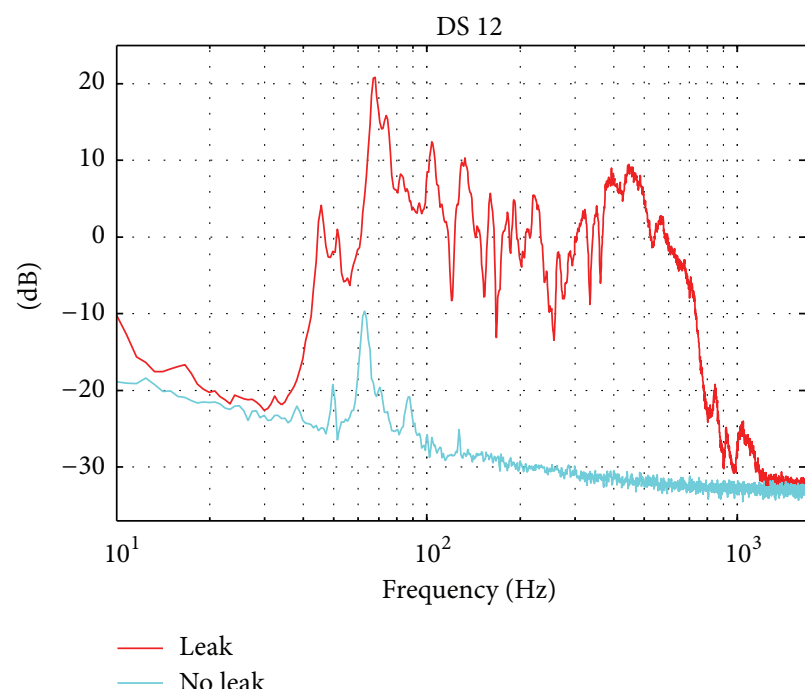

$(\mathrm{g})$

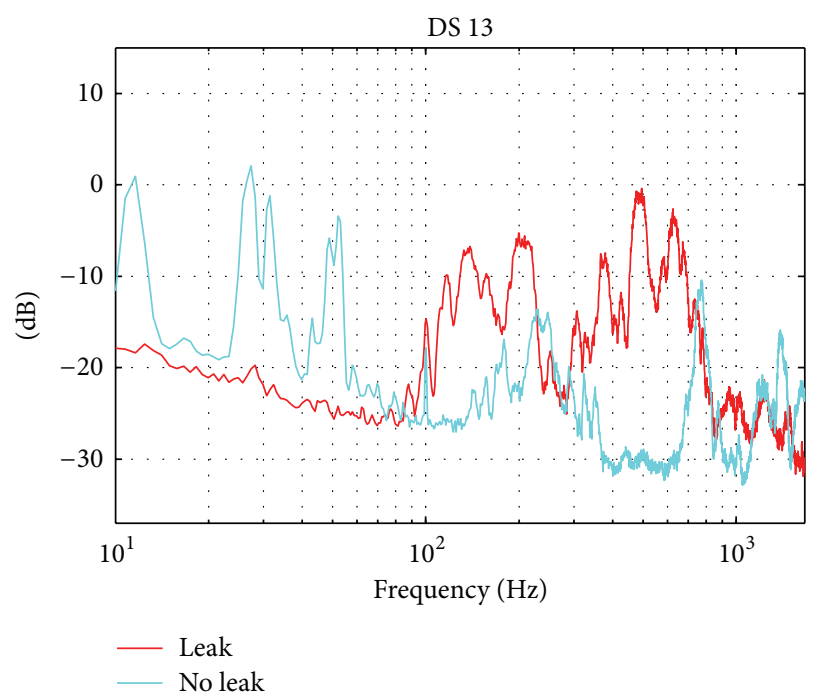

(h)

FIGURE 9: PSD of aggregated signals from datasets (a) DS 2, (b) DS 3, (c) DS 5, (d) DS 7, (e) DS 10, (f) DS 11, (g) DS 12 , and (h) DS 13.

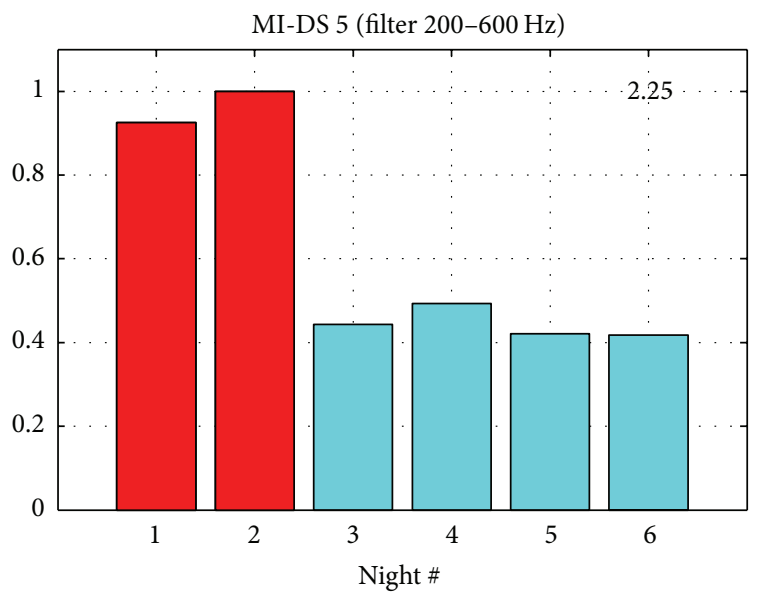

(a)

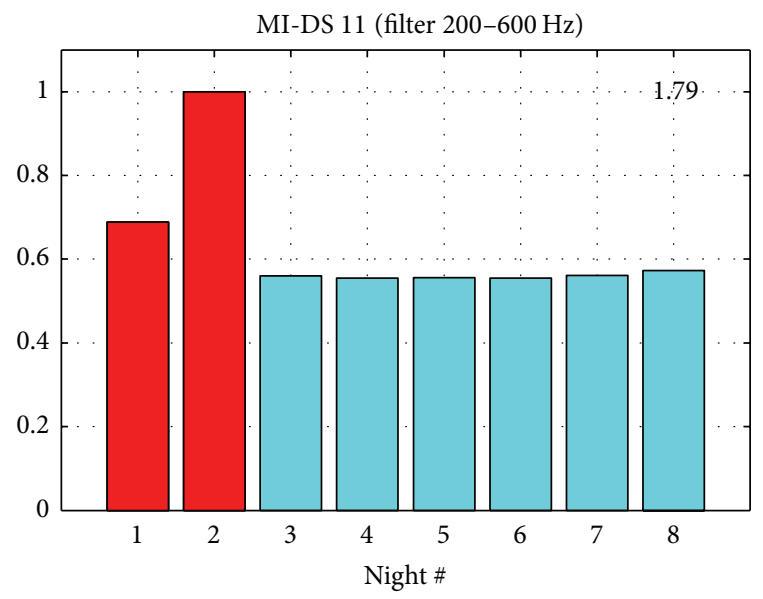

(b)

FIGURE 10: MI computed by the algorithm for filtered signals of datasets (a) DS 5, (b) DS 11.

Hence signal filtering proved a profitable solution for enhancing the algorithm sensitivity to leaks while keeping limited its complexity. In addition, the algorithm was confirmed to be capable of dealing with different issues possibly affecting measurements, such as transient environmental perturbations or saturation effects. The use of filters specifically tuned for plastic service pipes and the very limited sensitivity to the environmental noise represent a significant improvement over known available solutions for leak monitoring and detection. The proposed algorithm proved an effective and reliable monitoring tool for the piping systems of interest. Moreover it appears suitable to be implemented on an actual measuring device featuring rather simple and affordable electronics for achieving automatic leak detection.

The future steps of the research will aim at testing the detection algorithm on an enlarged database of real leaks, collected by setting up the prototypal measuring devices in some water districts of the network, thus starting a pilot leak detection program. The extended experimental campaign will make following the natural evolution of leaks over time possible, thus permitting to improve the measurement protocol and to further investigate the algorithm early detection capabilities.

\section{Conflict of Interests}

The authors declare that there is no conflict of interests regarding the publication of this paper.

\section{Acknowledgments}

This activity is performed in collaboration with Hera S.p.A. (Bologna, Italy), that is gratefully acknowledged for operative cooperation, use of facilities, and financial support. 


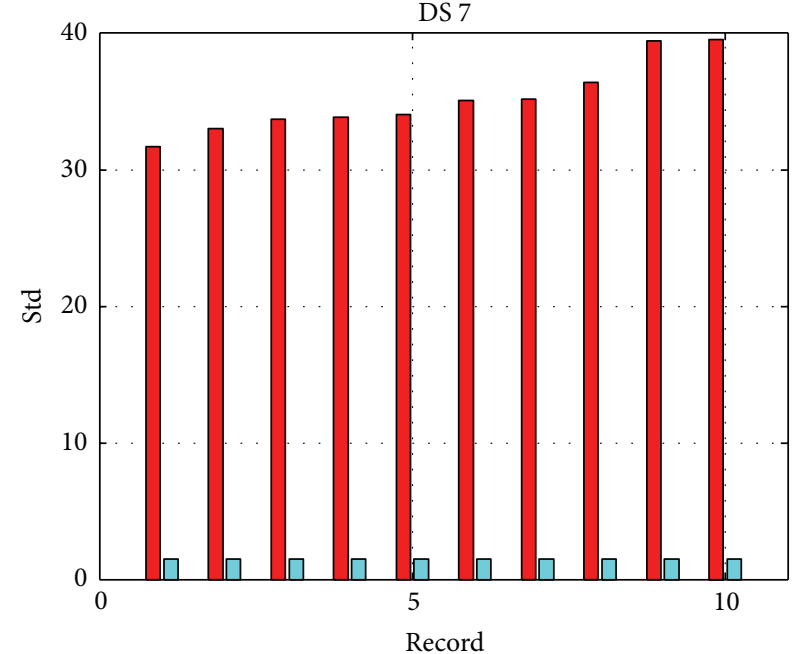

(a)

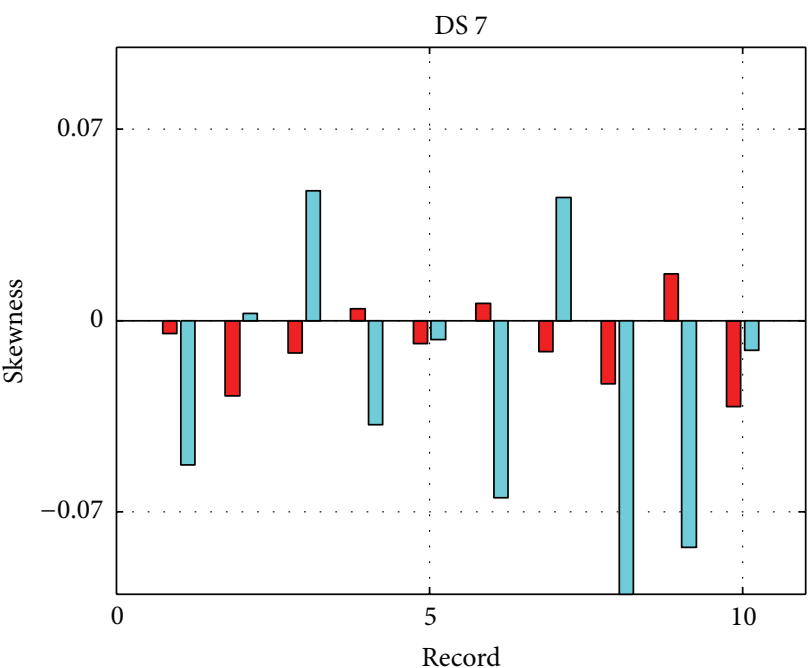

(b)

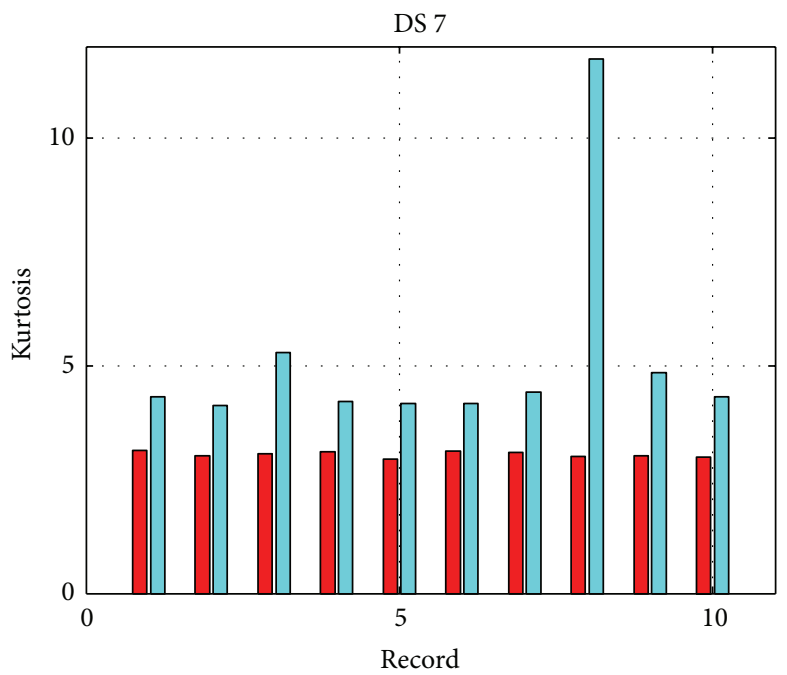

(c)

Figure 11: (a) STD, (b) skewness and (c) kurtosis values of the reduced subset for nights \#1 (leak, red) and \#2 (no leak, cyan) of DS 7.

\section{References}

[1] United States Environmental Protection Agency, "Control and mitigation of drinking water losses in distribution systems," Tech. Rep. EPA 816-R-10-019, 2010.

[2] BDEW-German Association of Energy and Water Industries, "VEWA Survey: Comparison of European Water and Waste water Prices," 2010, https://www.bdew.de.

[3] International Water Association, District Metered Areas Guidance Notes, International Water Association, London, UK, 2007.

[4] Y. A. Khulief, A. Khalifa, R. B. Mansour, and M. A. Habib, "Acoustic detection of leaks in water pipelines using measurements inside pipe," Journal of Pipeline Systems Engineering and Practice, vol. 3, no. 2, pp. 47-54, 2012.

[5] A. S. Papastefanou, P. F. Joseph, and M. J. Brennan, "Experimental investigation into the characteristics of in-pipe leak noise in plastic water filled pipes," Acta Acustica united with Acustica, vol. 98, no. 6, pp. 847-856, 2012.
[6] W. Li, W. Ling, S. Liu et al., "Development of systems for detection, early warning, and control of pipeline leakage in drinking water distribution: a case study," Journal of Environmental Sciences, vol. 23, no. 11, pp. 1816-1822, 2011.

[7] O. Hunaidi and A. Wang, "A new system for locating leaks in urban water distribution pipes," Management of Environmental Quality, vol. 17, no. 4, pp. 450-466, 2006.

[8] C. R. Fuller and F. J. Fahy, "Characteristics of wave propagation and energy distributions in cylindrical elastic shells filled with fluid," Journal of Sound and Vibration, vol. 81, no. 4, pp. 501-518, 1982.

[9] G. Pavić, "Vibroacoustical energy flow through straight pipes," Journal of Sound and Vibration, vol. 154, no. 3, pp. 411-429, 1992.

[10] J. M. Muggleton, M. J. Brennan, and R. J. Pinnington, "Wavenumber prediction of waves in buried pipes for water leak detection," Journal of Sound and Vibration, vol. 249, no. 5, pp. 939-954, 2003.

[11] M. J. Brennan, Y. Gao, and P. F. Joseph, "On the relationship between time and frequency domain methods in time delay 
estimation for leak detection in water distribution pipes," Journal of Sound and Vibration, vol. 304, no. 1-2, pp. 213-223, 2007.

[12] Y. Gao, M. J. Brennan, P. F. Joseph, J. M. Muggleton, and O. Hunaidi, "On the selection of acoustic/vibration sensors for leak detection in plastic water pipes," Journal of Sound and Vibration, vol. 283, no. 3-5, pp. 927-941, 2005.

[13] O. Hunaidi, W. Chu, A. Wang, and W. Guan, "Detecting leaks in plastic pipes," Journal/American Water Works Association, vol. 92, no. 2, pp. 82-94, 2000.

[14] M. Pal, N. Dixon, and J. Flint, "Detecting \& locating leaks in water distribution polyethylene pipes," in Proceedings of the World Congress on Engineering, vol. 2, London, UK, June-July 2010.

[15] O. Hunaidi and W. T. Chu, "Acoustical characteristics of leak signals in plastic water distribution pipes," Applied Acoustics, vol. 58, no. 3, pp. 235-254, 1999.

[16] A. Anastasopoulos, D. Kourousis, and K. Bollas, "Acoustic emission leak detection of liquid filled buried pipeline," Journal of Acoustic Emission, vol. 27, pp. 27-39, 2009.

[17] A. J. Brunner and M. Barbezat, "Acoustic emission monitoring of leaks in pipes for transport of liquid and gaseous media: a model experiment," Advanced Materials Research, vol. 13-14, pp. 351-356, 2006.

[18] S. J. Vahaviolos, R. K. Miller, D. J. Watts, V. V. Shemyakin, and S. A. Strizkov, "Detection and location of cracks and leaks in buried pipelines using acoustic emission," Journal of Acoustic Emission, vol. 19, pp. 172-183, 2001.

[19] T. Suzuki, Y. Ikeda, Y. Tomoda, and M. Ohtsu, "Water-leak evaluation of existing pipeline by acoustic emission," Journal of Acoustic Emission, vol. 23, pp. 272-276, 2005.

[20] M. F. Ghazali, S. B. M. Beck, J. D. Shucksmith, J. B. Boxall, and W. J. Staszewski, "Comparative study of instantaneous frequency based methods for leak detection in pipeline networks," Mechanical Systems and Signal Processing, vol. 29, pp. 187-200, 2012.

[21] N. Metje, P. R. Atkins, M. J. Brennan et al., "Mapping the Underworld - State-of-the-art review," Tunnelling and Underground Space Technology, vol. 22, no. 5-6, pp. 568-586, 2007.

[22] M. Bimpas, A. Amditis, and N. Uzunoglu, "Detection of water leaks in supply pipes using continuous wave sensor operating at $2.45 \mathrm{GHz}$," Journal of Applied Geophysics, vol. 70, no. 3, pp. 226-236, 2010.

[23] M. Fahmy and O. Moselhi, "Detecting and locating leaks in underground water mains using thermography," in Proceedings of the 26th International Symposium on Automation and Robotics in Construction (ISARC '09), pp. 61-67, Austin, Tex, USA, June 2009.

[24] A. Cataldo, R. Persico, G. Leucci et al., "Time domain reflectometry, ground penetrating radar and electrical resistivity tomography: a comparative analysis of alternative approaches for leak detection in underground pipes," NDT and E International, vol. 62, pp. 14-28, 2014.

[25] G. Leoni, C. Anzalone, D. Giunchi, and D. Nascetti, "Method for detecting the presence of leaks in a water distribution network and kit for applying the method," European Patent 2.107.357, 2009.

[26] A. Martini, M. Troncossi, A. Rivola, and D. Nascetti, "Preliminary investigations on automatic detection of leaks in water distribution networks by means of vibration monitoring," in Advances in Condition Monitoring of Machinery in NonStationary Operations, Lecture Notes in Mechanical Engineering, pp. 535-544, Springer, Berlin, Germany, 2014. 

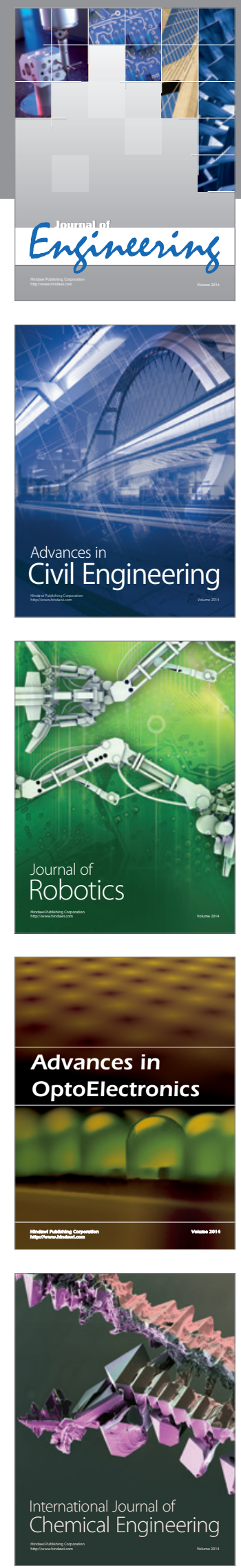

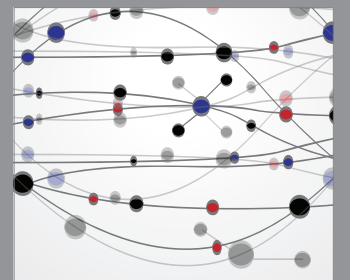

The Scientific World Journal
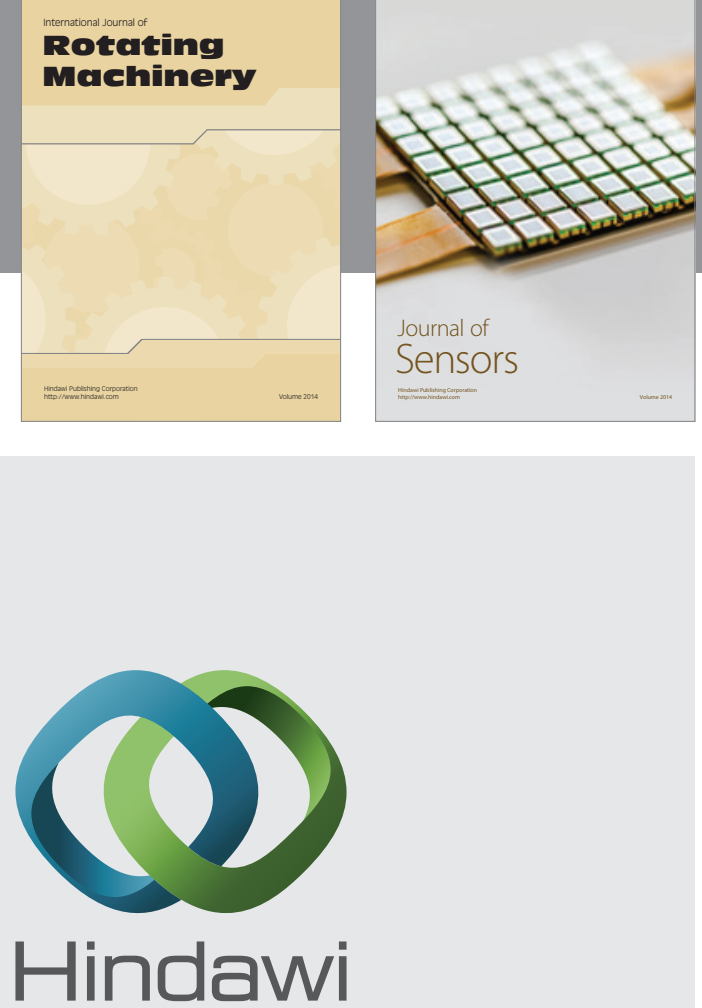

Submit your manuscripts at http://www.hindawi.com
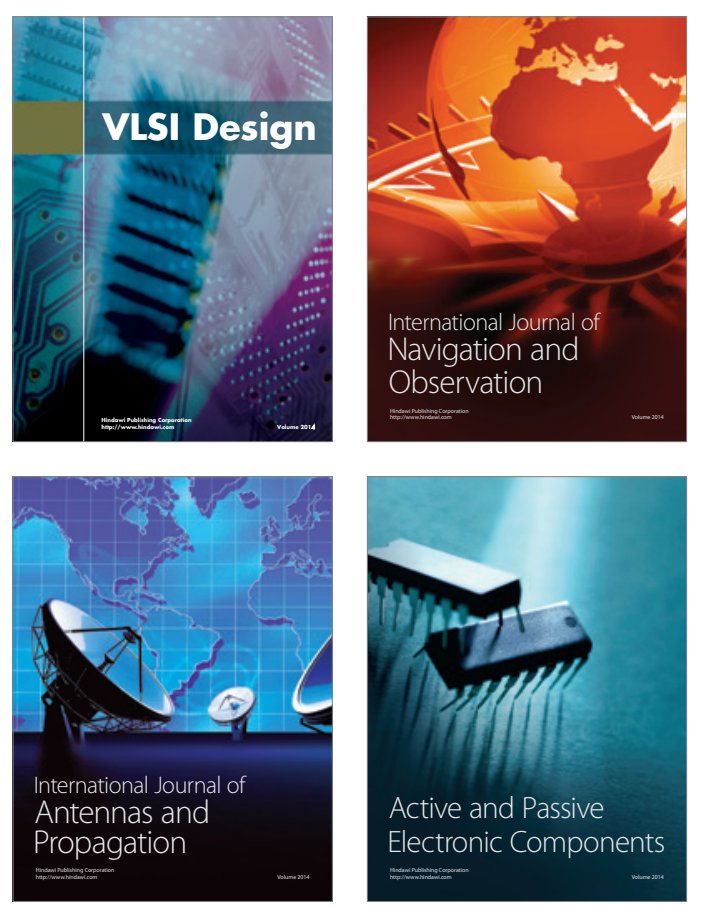
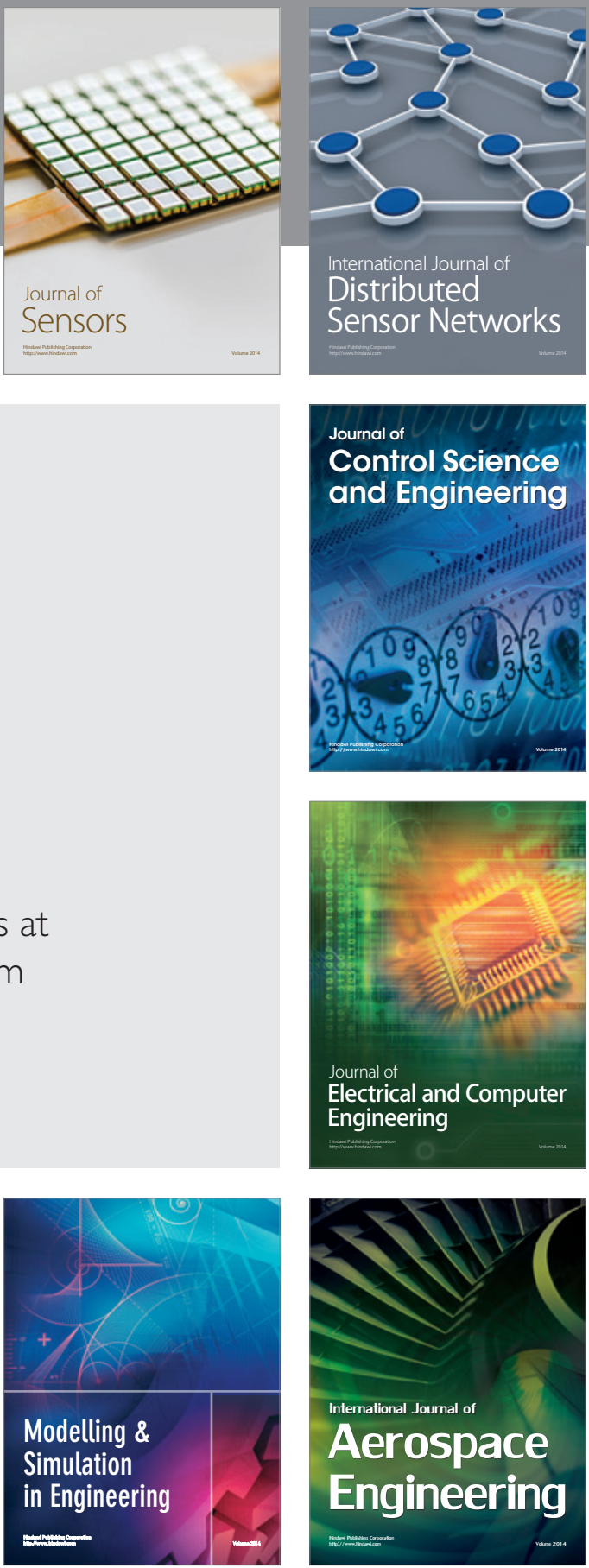

Journal of

Control Science

and Engineering
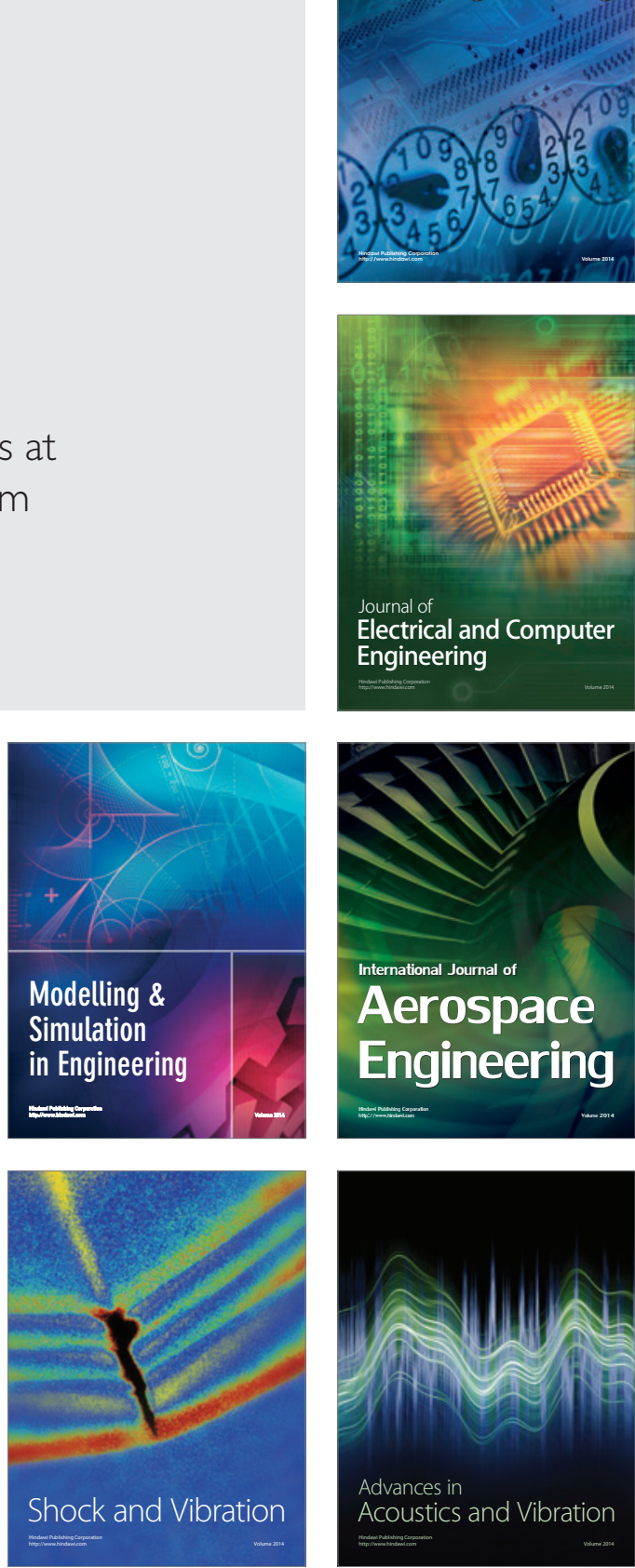